\title{
BIORREGULADORES PARA LA PRODUCCIÓN DE HIGO BAJO CONDICIONES DE INVERNADERO
}

\author{
BIOREGULATORS FOR FIG PRODUCTION UNDER \\ GREENHOUSE CONDITIONS
}

\author{
Alba Soberanes-Pérez ${ }^{1}$, Guillermo Calderón-Zavala ${ }^{1}{ }^{*}$, \\ Alfredo López-Jiménez ${ }^{1}$ y Horacio E. Alvarado-Raya ${ }^{2}$
}

\begin{abstract}
'Colegio de Postgraduados, Campus Montecillo, Postgrado en Recursos Genéticos y Productividad-Fruticultura, Texcoco, Estado de México, México. ²Universidad Autónoma Chapingo, Departamento de Preparatoria Agrícola, Chapingo, Texcoco, Estado de México, México.
\end{abstract}

\section{RESUMEN}

La demanda de higo (Ficus carica L.) fresco crece anualmente en el mercado nacional e internacional, y México tiene potencial para convertirse en importante productor mundial. Para lograrlo, es necesario incrementar el rendimiento, adelantar las cosechas y obtener frutos de calidad. La maduración escalonada de los siconos en la planta representa una desventaja económica, por lo que es importante concentrar la producción de higos maduros para reducir la frecuencia de cosechas. Se ha demostrado que los biorreguladores aceleran el desarrollo de los frutos y mejoran su rendimiento y calidad. El objetivo de esta investigación fue analizar el efecto de la aplicación de cinco combinaciones de biorreguladores (thidiazurón, ácido giberélico, ácido glutámico, triptófano y tiamina) sobre la brotación de yemas axilares secundarias, el crecimiento, rendimiento y calidad de frutos de yemas primarias. Como material genético se utilizaron higueras cv. Netzahualcóyotl cultivadas en invernadero. Los tratamientos se aplicaron por aspersión dirigida a las yemas axilares secundarias en reposo. Las variables evaluadas fueron brotación de yemas axilares secundarias, crecimiento y rendimiento del fruto de la yema principal. Al momento de cosecha se evaluó la longitud, diámetro y peso del fruto, color de la epidermis, firmeza, sólidos solubles totales y acidez titulable. Los biorreguladores adelantaron en $15 \mathrm{~d}$ el tiempo a la cosecha, excepto la combinación de ácido glutámico y tiamina. En los frutos se incrementó el contenido de sólidos solubles totales (5 a $\left.10^{\circ} \mathrm{Brix}\right)$, la longitud $(\approx 1 \mathrm{~cm})$, el diámetro $(\approx 0.5 \mathrm{~cm})$ y el peso individual $(5$ a $20 \mathrm{~g})$ con respecto al testigo. El color se afectó por los biorreguladores en forma negativa; los higos tuvieron zonas verdes e inmaduras cercanas al pedúnculo. El rendimiento de fruto no presentó diferencias signifiativas. Aunque los tratamientos con biorreguladores mejoraron algunas variables de calidad, ninguna combinación fue consistente para incrementar el rendimiento y la calidad de frutos de higo.

Palabras clave: Ficus carica, aminoácidos, biorreguladores, calidad de siconos, rendimiento.

\section{SUMMARY}

The demand for fresh fig (Ficus carica L.) grows annually in the national and international market, and Mexico has the potential to become a major world producer. To achieve this, it is necessary to increase the yield, advance the harvests and obtain quality fruits. The stepwise ripening of the syconia in the plant represents an economic disadvantage, so it is important to concentrate the production of ripe figs to reduce the frequency of harvests. It has been demonstrated that bioregulators accelerate the development of fruits and improve the yield and quality. The objective of this research was to analyze the effect of the application of five combinations of bioregulators (thidiazuron gibberellic acid, glutamic acid, tryptophan and thiamine) on the sprouting of secondary axillary buds, and growth, yield and quality of fruits of primary buds. Fig trees cv. Netzahualcoyotl grown under greenhouse conditions were used as genetic material. Treatments were applied by directed spraying on resting secondary axillary buds. The evaluated traits were secondary axillary bud sprouting, growth and yield of the main bud fruit; at harvest length, diameter and weight of the fruit, colour of the skin, firmness, total soluble solids and titratable acidity were evaluated. Bioregulators advanced the harvest time by 15 days, except for the combination of glutamic acid and thiamine. In fruits, the total soluble solids content ( 5 to $10^{\circ}$ Brix), length $(\approx 1 \mathrm{~cm})$, diameter $(\approx$ $0.5 \mathrm{~cm}$ ) and individual weight ( 5 to $20 \mathrm{~g}$ ) increased compared to the control. Color was affected by bioregulators in a negative way as figs had green and immature areas near the peduncle. Fruit yield did not show significant differences. Although bioregulatory treatments improved some quality traits, no combination was consistent to increase yield and quality of fruit figs.

Index words: Ficus carica, amino acids, bioregulators, quality of syconia, yield

\section{INTRODUCCIÓN}

En México, la higuera (Ficus carica L.) se ha cultivado desde la época de la colonia como una especie de traspatio (Muñoz et al., 2015); sin embargo, la demanda de higo fresco, como producto de exportación, es de 12 mil toneladas para el mercado de Estados Unidos de América (Macías et al., 2013). México tiene el potencial para convertirse en importante productor de higo; para lograrlo se requiere incrementar el rendimiento, adelantar las cosechas y obtener frutos de calidad. Una alternativa que ha funcionado en otros frutales es la aplicación de biorreguladores; éstos se definen como cualquier sustancia o microorganismo que se aplica a las plantas, en forma individual o mezclado con otro producto, con el objeto de mejorar la tolerancia al estrés e incrementar la calidad de las cosechas, entre otros beneficios (du Jardin, 2015). 
Los biorreguladores se aplican principalmente a cultivos de alto valor en invernadero y árboles frutales en campo abierto (Colla y Rouphael, 2015) como lichi (Litchi chinensis) (Zeng et al., 2012), manzana (Malus domestica) (Wójcik et al., 2016) y mango (Mangifera indica) (Gao et al., 2017; Osuna-Enciso et al., 2019). Entre los biorreguladores se encuentran el thidiazurón (TDZ), el ácido giberélico ( $\left.\mathrm{AG}_{3}\right)$, los aminoácidos y las vitaminas (Goyer, 2010; Kurubar et al., 2017; Nisler, 2018; Sadak et al., 2015). El TDZ, por su actividad citocinínica, estimula la división o expansión celular, mantiene la estructura de las clorofilas y rompe el letargo de yemas, entre otros efectos. La acción del TDZ en la planta depende de la concentración, momento de aplicación y especie tratada; adicionalmente, la interacción de los elementos mencionados con factores ambientales afecta, positiva o negativamente, la respuesta fisiológica (Nisler, 2018). Las concentraciones de aplicación son amplias, desde 2.5 hasta $500 \mathrm{mg} \mathrm{L}^{-1}$ en frutales (CalderónZavala y Rodríguez-Alcázar, 2000; Jung et al., 2015).

La aplicación de $\mathrm{AG}_{3}$ mejora la calidad y tamaño de frutos (Souza et al., 2010), actúa mediante la expansión celular (Li et al., 2011) y las concentraciones de aplicación varían entre especies. Los aminoácidos generan efectos positivos sobre el crecimiento y rendimiento de las plantas, son precursores de sustancias para la formación de metabolitos secundarios; además, mitigan los daños causados por estrés abiótico o biótico (Sadak et al., 2015; Yu et al., 2010).

Con la aplicación de ácido glutámico vía foliar existe la posibilidad de mejorar la asimilación de $\mathrm{N}$ en las plantas, lo que puede reflejarse en mayor rendimiento (Cao et al., 2010), capacidad fotosintética y acumulación de biomasa (Yu et al., 2010). El triptófano, según Mustafa et al. (2018), aumenta el rendimiento y contenido de nutrientes sin afectar la calidad de los cultivos; además, es precursor de auxinas. La aplicación de tiamina, o vitamina B1, mejora la resistencia de las plantas a factores estresantes y es cofactor enzimático en vías metabólicas como la glucólisis (Goyer, 2010). Como biorregulador, el $\mathrm{AG}_{3}$ se ha utilizado para adelantar hasta en 30 d la maduración y cosecha de frutos de higo en concentraciones de 20 a $120 \mathrm{mg} \mathrm{L}^{-1}$, y la reducción en el número de las cosechas representa una ventaja económica (Rodrigues et al., 1997). Por su parte, Kurubar et al. (2017) reportaron que la aplicación en diferente concentración y frecuencia de $\mathrm{AG}_{3}$ adelantó la cosecha de los higos, pero no incrementó el rendimiento.

En la higuera los siconos (frutos) aparecen de forma contínua, siempre se encuentran en las axilas de las hojas y normalmente sólo una yema axilar se trasforma en higo, mientras la otra puede dar lugar a una rama; en ocasiones, las dos yemas axilares pueden transformarse en frutos. Según la estación del año en que se producen los siconos, éstos se llaman brevas o higos. Las brevas pasan el invierno en reposo en las ramas de madera y se cosechan al año siguiente, mientras que los higos se desarrollan en las ramas de crecimiento del mismo año (Melgarejo, 2000). En el presente documento, para evitar confusiones, se denomina frutos de higo o higos a los siconos. Dado que los estudios sobre el uso de biorreguladores en higo son escasos, el objetivo de esta investigación fue analizar el efecto de la aplicación de cinco combinaciones de biorreguladores sobre la brotación de yemas axilares secundarias y el crecimiento, rendimiento y calidad de frutos de higo de las yemas primarias.

\section{MATERIALES Y MÉTODOS}

\section{Sitio experimental}

El sitio experimental se ubicó en el huerto San José del Colegio de Postgraduados, en Texcoco, Edo. de México, geográficamente localizado en las coordenadas $19^{\circ} 27^{\prime} 29^{\prime \prime}$ Ny $98^{\circ} 54^{\prime} 11^{\prime \prime}$ O, a una altitud de 2251 msnm. Las plantas se instalaron en un invernadero con cubierta plástica, tipo capilla simple, con techo semicircular de $3 \mathrm{~m}$ de altura y paredes verticales.

\section{Material vegetal y manejo agronómico}

El 19 de octubre 2016 se establecieron 60 plantas de higuera cv. Netzahualcóyotl provenientes de estacas de madera dura y tres meses de enraizamiento previo. Se utilizaron macetas de $38 \mathrm{~L}$ (una planta por maceta) con sustrato a base de vermiculita, turba, suelo del huerto San José y compost de estiércol bovino, en proporciones 1:1:1:3 en base a volumen. La distancia entre las macetas fue de $60 \mathrm{~cm}$ de centro a centro y $40 \mathrm{~cm}$ entre hileras. En febrero de 2017 las plantas se podaron a tres ramas, se dejaron las de mismo vigor y tamaño, y se mantuvieron durante todo el ciclo de producción. El manejo de las plantas incluyó riego cada tercer día (3 L/maceta) desde la plantación hasta marzo 2017; después, $4 \mathrm{~L}$ diarios en el periodo de abril a julio 2017. Para la fertilización se aplicaron $2 \mathrm{~g} /$ maceta, cada $15 \mathrm{~d}$, de una mezcla fertilizante que contenía N (18 $\%), \mathrm{P}(18 \%), \mathrm{K}(18 \%), \mathrm{S}(0.8 \%), \mathrm{Mg}(0.5 \%), \mathrm{B}\left(100 \mathrm{mg} \mathrm{kg}^{-1}\right)$, $\mathrm{Cu}\left(100 \mathrm{mg} \mathrm{kg}^{-1}\right), \mathrm{Fe}\left(400 \mathrm{mg} \mathrm{kg}^{-1}\right), \mathrm{Mn}\left(200 \mathrm{mg} \mathrm{kg}^{-1}\right), \mathrm{Mo}$ (100 $\mathrm{mg} \mathrm{kg}^{-1}$ ) y Zn (100 $\left.\mathrm{mg} \mathrm{kg}^{-1}\right)$; adicionalmente, se aplicó $1 \mathrm{~g} /$ maceta, cada 15 días, de una mezcla fertilizante que contenía N (12\%), $\mathrm{P}_{2} \mathrm{O}_{5}(11 \%), \mathrm{K}_{2} \mathrm{O}(18 \%), \mathrm{MgO}(2.7 \%), \mathrm{S}(8$ $\%), F e(0.2 \%), B\left(150 \mathrm{mg} \mathrm{kg}^{-1}\right), \mathrm{Mn}\left(200 \mathrm{mg} \mathrm{kg}^{-1}\right)$ y Zn (200 $\left.\mathrm{mg} \mathrm{kg}^{-1}\right)$. Durante todo del ciclo de producción se aplicó: $\mathrm{N}$, 15.1 g; P, 13.8 g; K,15.2 g; Mg, 0.7 g; S, 2 g; B, 10 mg; Fe, 65 mg; Mn y Zn, 18 mg; y Co y Mo, 7.2 mg. 


\section{Tratamientos}

Los biorreguladores se aplicaron en la etapa reproductiva (Ikegami et al., 2013) el 5 de marzo de 2017 y se repitieron después de 15 d (19 de marzo). Los tratamientos consistieron en: T1 (100 mg L-1 TDZ y $50 \mathrm{mg} \mathrm{L}^{-1} \mathrm{AG}_{3}$ ); T2 (100 $\mathrm{mg} \mathrm{L}^{-1} \mathrm{TDZ}, 25 \mathrm{mg} \mathrm{L}^{-1} \mathrm{AG}_{3}$ y $150 \mathrm{mg} \mathrm{L}^{-1}$ triptófano); T3 (50 mg L-1 TDZ, $25 \mathrm{mg} \mathrm{L}^{-1} \mathrm{AG}_{3}$ y $150 \mathrm{mg} \mathrm{L}^{-1}$ ácido glutámico); T4 (150 mg L-1 ácido glutámico y $100 \mathrm{mg} \mathrm{L}^{-1}$ tiamina); T5 (25 mg L-1 TDZ, $150 \mathrm{mg} \mathrm{L}^{-1}$ triptófano y 150 mg L ${ }^{-1}$ ácido glutámico) y T6 (testigo, sin aplicación). Las fuentes fueron: TDZ $\left(\mathrm{C}_{9} \mathrm{H}_{8} \mathrm{~N}_{4} \mathrm{OS}\right) 42.4 \%$, ácido giberélico $\left(\mathrm{C}_{19} \mathrm{H}_{22} \mathrm{O}_{6}\right) 90 \%$, tiamina $\left(\mathrm{C}_{12} \mathrm{H}_{17} \mathrm{~N}_{4} \mathrm{OS}^{+}\right)$, ácido glutámico $\left(\mathrm{C}_{5} \mathrm{H}_{9} \mathrm{NO}_{4} \cdot \mathrm{HCl}\right)$ y triptófano $\left(\mathrm{C}_{11} \mathrm{H}_{12} \mathrm{~N}_{2} \mathrm{O}_{2}\right) \quad 100 \%$ Se adicionaron $0.5 \mathrm{~mL}$ de INEX-A $\AA$ por $L$ de tratamiento como surfactante. Las aspersiones se dirigieron a las yemas axilares secundarias en reposo de los nudos de cada rama; es decir, a las yemas adyacentes al fruto principal, hasta el punto de escurrimiento. Para la aplicación se utilizó un atomizador por solución.

\section{Variables y frecuencia de medición}

Para el porcentaje de brotación de yemas secundarias, el día anterior a la aplicación de los tratamientos se seleccionó una rama al azar por planta y se contaron las yemas axilares secundarias (adyacentes al fruto principal) en reposo; después de la aplicación de los tratamientos se cuantificaron las yemas axilares secundarias brotadas cada siete y hasta 30 días después de las aspersiones.

Para generar la curva de crecimiento de frutos de higo se midió con un vernier (Truper®, China) el diámetro ecuatorial en cinco higos seleccionados al azar de cinco plantas por tratamiento; el primer dato se registró cuando los frutos alcanzaron $5 \mathrm{~mm}$ de diámetro, y después, cada 15 días a partir de la primera medición.

En el periodo de mayo a julio 2017 se cosecharon semanalmente los frutos de higo en madurez de consumo, los datos se reportaron quincenalmente. La longitud se midió desde el pedúnculo del higo hasta el ostiolo, y el diámetro máximo se determinó en la sección ecuatorial y los valores obtenidos se registraron en $\mathrm{cm}$. El peso en g se determinó con una balanza digital Santulß 6927 (Ningbo Mini Tree Co., Ltd., Jiangnan, China). El rendimiento de fruto se determinó con el peso (kg/planta) y se obtuvo el número de frutos por planta. La calidad de los higos se evaluó una vez al mes durante la producción utilizando cinco frutos por tratamiento. El color de la epidermis se midió en la zona ecuatorial de los higos en dos lados opuestos; se usó un colorímetro portátil Colorimeter NR20XE (3nh® Technology Co., Ltd., Nanshan, China), y se registraron los valores de luminosidad $(L)$, coordenada cromática $a *$, coordenada cromática b*, chroma (C) y ángulo de tono Hue ${ }^{\circ}$ (Yemiş et al., 2012). La firmeza se determinó con un texturómetro digital Modelo FDV30 (Wagner Instruments $\AA$, Cambridgeshire, Inglaterra) provisto de un puntal cónico de $7 \mathrm{~mm}$; se registró la fuerza necesaria para penetrar la epidermis en la zona ecuatorial en dos lados opuestos del fruto, los datos se reportaron en newtons (N) (García et al., 2015). El contenido de sólidos solubles totales (SST) se midió por refractometría, de acuerdo con la AOAC (1990), se utilizó un refractómetro digital modelo PR-32a (Atagoß, Tokio, Japón), al cual se le colocó una gota de jugo obtenido de la parte central del higo, los valores se expresaron en ${ }^{\circ}$ Brix. La acidez titulable (AT) se determinó por el método de AOAC (1990); en un recipiente se pesaron $5 \mathrm{~g}$ de pulpa de higo y se licuaron con $40 \mathrm{~mL}$ de agua destilada, de la mezcla obtenida $(41 \mathrm{~mL})$, se tomaron $20 \mathrm{~mL}$ y se agregaron tres gotas de fenolftaleína, esta mezcla se tituló con $\mathrm{NaOH} 0.01 \mathrm{~N}$ hasta lograr el vire a color púrpura; la acidez titulable (AT) se expresó como porcentaje de ácido cítrico en la pulpa.

\section{Diseño experimental y análisis estadístico}

El experimento se instaló bajo un diseño completamente al azar con 10 repeticiones, la unidad experimental fue una planta con tres ramas por maceta. El análisis de datos incluyó prueba de normalidad (Anderson-Darling), de homogeneidad de varianzas (Prueba de Bartlett), análisis de varianza y prueba de comparación de medias de Tukey $(P \leq 0.05)$. Los análisis se realizaron con el paquete estadístico SASß 9.1. (SAS Institute, 2004).

\section{RESULTADOS Y DISCUSIÓN}

\section{Porcentaje de brotación de yemas axilares secundarias}

Los tratamientos T1 y T2 promovieron la brotación de yemas secundarias vegetativas en $100 \%$; no se presentaron yemas reproductivas. Con T3 y T5 la brotación de yemas reproductivas fue de 1.3 y $2.7 \%$ respectivamente, el resto fueron vegetativas, mientras que con T4 y T6 las yemas secundarias permanecieron en reposo. Por otro lado, ambos tipos de brotes presentaron clorosis y necrosis; las yemas reproductivas fueron más susceptibles, pues se desprendieron a los 20 días después de la aplicación (DDA) de los biorreguladores y las vegetativas a los 25 DDA. Con el T5 se presentó el mayor porcentaje de brotación de yemas secundarias reproductivas; estos resultados se atribuyen al TDZ en los tratamientos; este biorregulador rompe el letargo de yemas (Nisler, 2018), tanto reproductivas como vegetativas (Zermeño et al., 2010); sin embargo, en dosis altas ocasiona fitotoxicidad (Calderón-Zavala y Rodríguez-Alcázar, 2000). Se ha reportado que las giberelinas antagonizan con la actividad citocinínica del 
TDZ; por otro lado, su combinación puede ser ventajosa en concentraciones apropiadas (Nisler, 2018); en este sentido, con el T3 se obtuvo un importante porcentaje de brotación de yemas secundarias reproductivas. $E l \mathrm{AG}_{3}$ rompe el letargo; no obstante, utiliza los carbohidratos para el crecimiento vegetativo en lugar del desarrollo de los frutos en higuera Poona (Kurubar et al., 2017). Los aminoácidos (Sadak et al., 2015) y la tiamina (Belal et al., 2016) tienen efectos positivos sobre las plantas, pero al parecer no actúan sobre la emisión de yemas.

No se presentaron frutos de las yemas axilares secundarias tratadas con los biorreguladores; por lo tanto, se analizó el efecto de los biorreguladores en los frutos de la yema principal en las siguientes variables.

\section{Curva de crecimiento de fruto}

La Figura 1 muestra que el crecimiento de los frutos de higo Netzahualcóyotl de las yemas principales se incrementó después de la primera aplicación (5 de marzo). A los 10 DDA el diámetro de los frutos fue $15 \mathrm{~mm}$ mayor con T1, T2, T3 y T5 comparado con el testigo (T6) y T4. El crecimiento de los higos fue lento del 15 al 30 de marzo y se aceleró a partir del 15 de abril; sin embargo, los frutos del testigo y T4 no tuvieron el mismo comportamiento. Estos resultados concuerdan con los obtenidos por Erogul y Sen (2015), donde aplicaciones con $\mathrm{AG}_{3}$ en ciruelo (Prunus domestica), TDZ en litchi (Zeng et al., 2012), triptófano en granado (Punica granatum) (El Sayed et al., 2014) y ácido glutámico en lima mexicana (Citrus aurantifolia) (Ariza et al., 2015), en concentraciones similares, incrementaron el diámetro de los frutos cuando se asperjaron en épocas tempranas de crecimiento.

El crecimiento en diámetro de los frutos de higo Netzahualcóyotl se atribuye al efecto del $\mathrm{AG}_{3}$ y TDZ en los tratamientos, pues ambos biorreguladores han demostrado ser efectivos para este fin; el primero causa expansión o agrandamiento celular (Li et al., 2011) y el segundo, por su actividad citocinínica, estimula la división o expansión celular (Nisler, 2018). Los aminoácidos mejoran la asimilación del N (Cao et al., 2010) y la tiamina reduce el estrés de las plantas (Goyer, 2010), lo que puede reflejarse en frutos de mayor diámetro; sin embargo, en conjunto (T4) no favorecieron el crecimiento. Luego de la segunda aplicación (19 de marzo) no se observó incrementó en el diámetro. La repetida aplicación de biorreguladores no garantiza efectos positivos (Kurubar et al., 2017).

Por último, el crecimiento de los frutos de higo Netzahualcóyotl coincide con una curva doble sigmoide reportada para esta especie (Bahar y Lichter, 2018). La fase de transición (II) se redujo en 15 d y con ello adelantó la cosecha de los frutos con T1, T2, T3 y T5 con respecto al testigo y T4, lo que permite concentrar las cosechas, reducir el número de éstas y alcanzar precios altos por producción adelantada.

\section{Longitud, diámetro y peso de fruto}

Se encontró que los frutos de las plantas tratadas con biorreguladores, en general, fueron de mayor longitud, diámetro y peso, comparados con los frutos del testigo (T6). Los frutos cosechados el 1 de mayo (Cuadro 1) no presentaron diferencia estadística en longitud; en cosechas posteriores y hasta el mes de junio, los frutos de T1, T2, T3 y T5 fueron $1 \mathrm{~cm}$ más largos con respecto al testigo. En la cosecha del 16 de mayo al 16 de junio, el diámetro de los frutos de T1, T2 y T3 fue al menos $0.5 \mathrm{~cm}$ mayor en comparación con el testigo (T6). Los frutos de higo tratados con biorreguladores obtuvieron mayor peso con respecto al testigo; ésto fue evidente en la cosecha del 16 de junio, donde los frutos de T1, T2, T3 y T5 superaron al T4 y al testigo en $20 \mathrm{~g}$ (Cuadro 2). Por último, el T4 no favoreció el incremento en longitud, diámetro y peso de los higos.

El incremento en longitud, diámetro y peso de los frutos de higuera que recibieron la aplicación de biorreguladores coinciden con los reportados en otras especies, donde aspersiones de TDZ en peral (Pyrus communis) (Hawerroth et al., 2011), $A G_{3}$ en piña (Ananas comosus) (Li et al., 2011) y la combinación de éstos en arándano (Vaccinium ashei) (Zang et al., 2016) incrementaron el tamaño (longitud y diámetro) o peso de los frutos. Al respecto, el TDZ estimula la división y expansión celular en las primeras etapas del desarrollo (Nisler, 2018) y el $\mathrm{AG}_{3}$ ocasiona expansión o agrandamiento celular (Li et al., 2011). El ácido glutámico incrementó el diámetro en frutos de lima mexicana (Ariza et al., 2015) y el triptófano en granada (El Sayed et al., 2014), al mejorar la asimilación del $\mathrm{N}$ en las plantas (Cao et al., 2010; Mustafa et al., 2018). Con relación a la tiamina, se reporta que ésta reduce el estrés en las plantas (Goyer, 2010). Como se puede observar, con la aplicación de los biorreguladores se tienen respuestas inconsistentes (Milić et al., 2018). Al respecto, Kurubar et al. (2017) indicaron que higueras Poona asperjadas tres veces con $60 \mathrm{mg} \mathrm{L}^{-1}$ de $\mathrm{AG}_{3}$ en intervalos de $15 \mathrm{~d}$ al inicio de la brotación de las yemas dieron como resultado frutos más largos, pero con diámetro y peso reducidos; esto último representa una desventaja económica que se refleja en bajo rendimiento. La interacción positiva o negativa de los biorreguladores depende del proceso biológico de la planta, tejido, edad, etapa de desarrollo, condiciones ambientales, concentración, composición y frecuencia de aplicación (Kurubar et al., 2017; Weiss y Ori, 2007). Finalmente, los frutos de higo de mayor tamaño y peso son de interés 


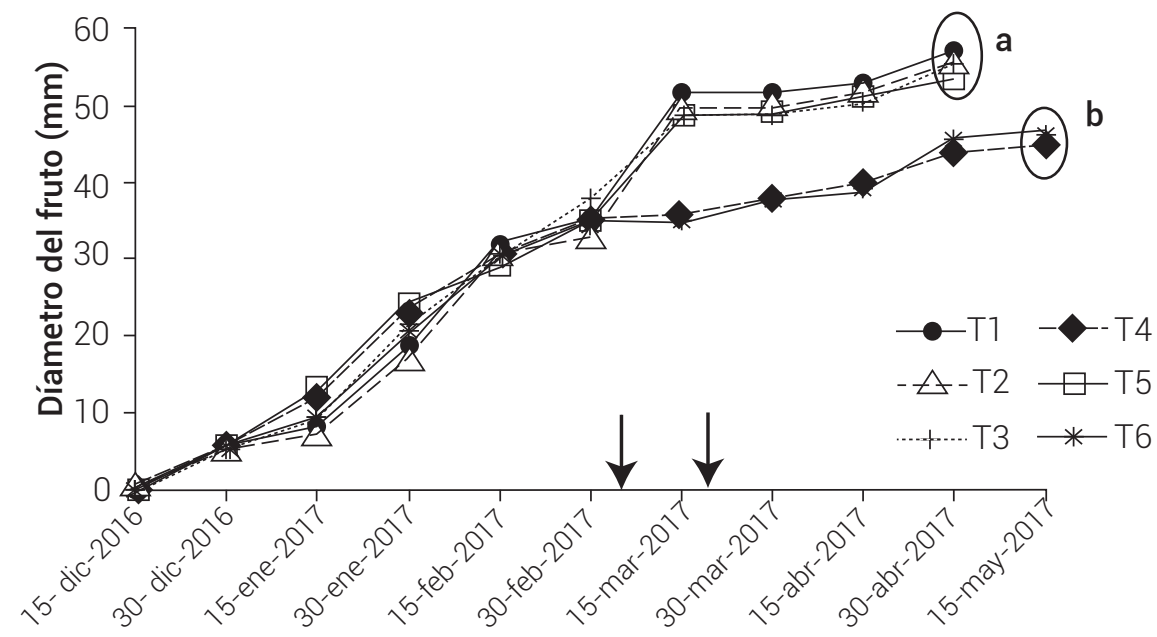

Fechas de aplicación

Figura 1. Curva de crecimiento de frutos de higo cv. Netzahualcóyotl. Las flechas indican la aplicación de los biorreguladores.

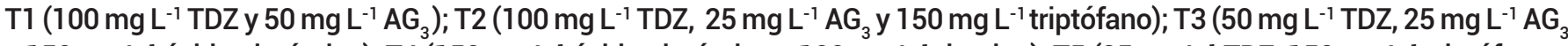
y $150 \mathrm{mg} \mathrm{L}^{-1}$ ácido glutámico); T4 (150 mg L-1 ácido glutámico y $100 \mathrm{mg} \mathrm{L}^{-1}$ tiamina); T5 (25 mg L-1 TDZ, $150 \mathrm{mg} \mathrm{L}^{-1}$ triptófano y $150 \mathrm{mg} \mathrm{L}^{-1}$ ácido glutámico) y T6 (testigo, sin aplicación). Diferentes letras en el gráfico indican diferencia estadística $(P \leq 0.05)$.

Cuadro 1. Longitud y diámetro $(\mathrm{cm})$ de los frutos de higo cv. Netzahualcóyotl tratados con biorreguladores y cosechados en diferentes fechas durante 2017.

\begin{tabular}{|c|c|c|c|c|c|c|c|c|c|c|}
\hline \multirow{2}{*}{ Tratamiento } & \multicolumn{2}{|c|}{1 de mayo } & \multicolumn{2}{|c|}{16 de mayo } & \multicolumn{2}{|c|}{1 de junio } & \multicolumn{2}{|c|}{16 de junio } & \multicolumn{2}{|c|}{1 de julio } \\
\hline & $\mathrm{L}$ & $\mathrm{D}$ & $\mathrm{L}$ & $\mathrm{D}$ & $\mathrm{L}$ & $\mathrm{D}$ & $\mathrm{L}$ & $\mathrm{D}$ & $\mathrm{L}$ & $\mathrm{D}$ \\
\hline $\mathrm{T} 1$ & $4.8 \mathrm{a}$ & $3.6 \mathrm{~b}$ & 5.9 a & $4.5 \mathrm{a}$ & $6.4 \mathrm{a}$ & $5.3 a$ & $6.0 \mathrm{a}$ & $5.5 a$ & $5.9 a$ & $5.5 a$ \\
\hline T2 & $5.0 \mathrm{a}$ & $3.8 a b$ & $5.8 \mathrm{a}$ & $4.6 \mathrm{a}$ & $6.3 \mathrm{a}$ & $5.4 \mathrm{a}$ & $6.1 \mathrm{a}$ & $5.7 \mathrm{a}$ & $6.1 \mathrm{a}$ & $5.7 \mathrm{a}$ \\
\hline T3 & $5.1 \mathrm{a}$ & $3.8 a b$ & $5.7 \mathrm{a}$ & $4.5 \mathrm{a}$ & $6.1 \mathrm{a}$ & $5.5 \mathrm{a}$ & $5.9 a$ & $5.8 \mathrm{a}$ & $5.6 a$ & $5.6 a$ \\
\hline T4 & $4.9 a$ & $3.8 a b$ & $5.3 b$ & $4.1 \mathrm{c}$ & $5.4 \mathrm{c}$ & $4.4 \mathrm{C}$ & $5.4 b$ & $4.6 \mathrm{~b}$ & $5.6 a$ & $4.7 b$ \\
\hline T5 & $5.1 \mathrm{a}$ & $4.0 \mathrm{a}$ & $5.4 \mathrm{~b}$ & $4.3 b$ & $6.0 \mathrm{ab}$ & $4.9 b$ & $6.0 \mathrm{a}$ & $5.6 a$ & $5.8 \mathrm{a}$ & $5.6 a$ \\
\hline T6 & $4.7 \mathrm{a}$ & $3.8 a b$ & $4.9 \mathrm{c}$ & $3.8 \mathrm{c}$ & $5.6 \mathrm{bc}$ & $4.5 \mathrm{bc}$ & $5.2 b$ & $4.6 \mathrm{~b}$ & $5.5 \mathrm{a}$ & $4.7 b$ \\
\hline CV (\%) & 12.5 & 10.2 & 7.4 & 7.3 & 9.0 & 11.9 & 8.1 & 9.2 & 9.3 & 10.5 \\
\hline
\end{tabular}

Medias con letras iquales en las columnas no son estadísticamente diferentes (Tukey, P $\leq 0.05)$. T1 (100 mg L-1 TDZ y $50 \mathrm{mg} \mathrm{L}^{-1} \mathrm{AG}$ ); T2 (100 mg $\mathrm{L}^{-1} \mathrm{TDZ}, 25 \mathrm{mg} \mathrm{L}^{-1} \mathrm{AG}_{3}$ y $150 \mathrm{mg} \mathrm{L}^{-1}$ triptófano); T3 (50 mg L-1 TDZ, $25 \mathrm{mg} \mathrm{L}^{-1} \mathrm{AG}_{3}$ y $150 \mathrm{mg} \mathrm{L}^{-1}$ ácido glutámico); T4 (150 mg L-1 ácido glutámico y $100 \mathrm{mg} \mathrm{L}^{-1}$ tiamina); T5 (25 mg L-1 TDZ, $150 \mathrm{mg} \mathrm{L}^{-1}$ triptófano y $150 \mathrm{mg} \mathrm{L}^{-1}$ ácido glutámico) y T6 (testigo, sin aplicación). L: Iongitud, D: diámetro, CV: coeficiente de variación.

Cuadro 2. Peso promedio de fruto (g) de los higos cv. Netzahualcóyotl tratados con biorreguladores y cosechados en diferentes fechas durante 2017.

\begin{tabular}{llllll}
\hline Tratamiento & 1 mayo & 16 mayo & 1 junio & 16 junio & 1 julio \\
\hline T1 & $31.5 \mathrm{~b}$ & $39.5 \mathrm{ab}$ & $71.0 \mathrm{a}$ & $81.1 \mathrm{a}$ & $58.2 \mathrm{ab}$ \\
T2 & $31.6 \mathrm{~b}$ & $43.1 \mathrm{a}$ & $66.3 \mathrm{a}$ & $80.8 \mathrm{a}$ & $68.1 \mathrm{a}$ \\
T3 & $40.1 \mathrm{a}$ & $42.5 \mathrm{a}$ & $69.5 \mathrm{a}$ & $84.0 \mathrm{a}$ & $62.0 \mathrm{ab}$ \\
T4 & $36.2 \mathrm{ab}$ & $38.1 \mathrm{bc}$ & $46.6 \mathrm{c}$ & $59.6 \mathrm{~b}$ & $53.2 \mathrm{~b}$ \\
T5 & $36.0 \mathrm{ab}$ & $41.0 \mathrm{ab}$ & $55.7 \mathrm{~b}$ & $82.3 \mathrm{a}$ & $53.8 \mathrm{a}$ \\
T6 & $32.4 \mathrm{~b}$ & $35.0 \mathrm{c}$ & $45.0 \mathrm{c}$ & $57.1 \mathrm{~b}$ & $52.1 \mathrm{~b}$ \\
CV (\%) & 14.7 & 15.4 & 25.2 & 15.7 & 24.6 \\
\hline
\end{tabular}

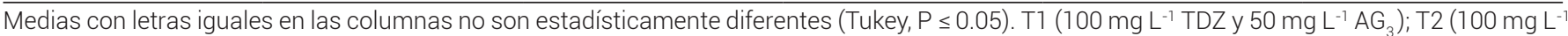
TDZ, $25 \mathrm{mg} \mathrm{L}^{-1} \mathrm{AG}_{3}$ y $150 \mathrm{mg} \mathrm{L}^{-1}$ triptófano); T3 (50 $\mathrm{mg} \mathrm{L}^{-1} \mathrm{TDZ}, 25 \mathrm{mg} \mathrm{L}^{-1} \mathrm{AG}_{3}$ y $150 \mathrm{mg} \mathrm{L}^{-1}$ ácido glutámico); T4 (150 mg L-1 ácido glutámico y 100 $\mathrm{mg} \mathrm{L}^{-1}$ tiamina); T5 (25 mg L-1 TDZ, $150 \mathrm{mg} \mathrm{L}^{-1}$ triptófano y $150 \mathrm{mg} \mathrm{L}^{-1}$ ácido glutámico) y T6 (testigo, sin aplicación). CV: coeficiente de variación. 
para los productores debido a que éstos reciben un mejor precio en el mercado (Pereira et al., 2017).

\section{Rendimiento y número de frutos}

Con respecto al rendimiento, no hubo diferencias estadísticas significativas entre tratamientos, con valores de 1.28 a $1.62 \mathrm{~kg} /$ planta. Estos resultados coinciden con los obtenidos por Kurubar et al. (2017) en higuera Poona, donde la concentración y frecuencia de aspersión con $\mathrm{AG}_{3}$ no modificaron el rendimiento; sin embargo, algunas variables de calidad mejoraron. En cuanto al número de frutos, se presentaron diferencias significativas; el T4 y testigo fueron los mejores tratamientos (30 frutos/planta), mientras que con T1, T2, T3 y T5 existió una tendencia a disminuir el número de frutos/planta; T2 obtuvo 22 frutos/ planta y resultó el peor tratamiento. Lo anterior indica que las combinaciones de $T D Z, A_{3}$ y los aminoácidos (triptófano y ácido glutámico) afectan de forma negativa la cantidad de frutos/planta. Al respecto, Hawerroth et al. (2011) encontraron que aspersiones de TDZ y $\mathrm{AG}_{3}$ en concentraciones de $20 \mathrm{mg} \mathrm{L}^{-1}$ estimulan el amarre de frutos en peral, pero también aumentan la competencia por fotoasimilados entre frutos de la misma inflorescencia y, en general, ocasionan la absición de los frutos más pequeños. Lo anterior permite inferir que las higueras $\mathrm{cv}$. Netzahualcóyotl que recibieron los tratamientos con TDZ, $\mathrm{AG}_{3} \mathrm{O}$ ambos, en concentraciones altas, abortaron los frutos de menor vigor, mientras que el T4 no afectó esta variable.

\section{Color de la epidermis}

El color en frutos de higo indica madurez y calidad (Crisosto et al., 2010). Los tratamientos afectaron el color de los frutos. Los valores a* de los frutos de T1 (17.4) y T5 (19.2) superaron al testigo (10.9) y desarrollaron un color púrpura, mientras que los frutos del testigo mostraron tendencia al color rojo. Con respecto al chroma (C), los frutos de T1 (18.5), T2 (18.5) y T5 (19.7) presentaron mayor saturación, comparados con los del testigo (11.8). Los valores de color en la epidermis obtenidos en esta investigación coinciden con los reportados para higos cosechados en madurez de consumo (Crisosto et al., 2010). El color de los higos no fue homogéneo, la zona cercana al pedúnculo permaneció verde en los frutos de T1, T2, T3 y T5 (Figura 2). La presencia de secciones verdes en los frutos se debe a la aplicación de cuatro compuestos: $T D Z, A_{3}$, ácido glutámico y triptófano. El TDZ mantiene las concentraciones de clorofila en los tejidos (Nisler, 2018); se ha señalado que el TDZ de 1 a $5 \mathrm{mg} \mathrm{L}^{-1}$ en lichi retrasó la coloración de los frutos y disminuyó el contenido de antocianinas (Zeng et al., 2012); además, Ariza et al. (2015) reportaron que la aplicación de $1 \mathrm{mg} \mathrm{L}^{-1}$ de la citocinina sintética mejoró el color verde en lima mexicana. En este sentido, la aspersión de $\mathrm{AG}_{3}$ en concentraciones de $30 \mathrm{a}$ $60 \mathrm{mg} \mathrm{L}^{-1}$ en cerezo (Prunus cerasus) retrasó la síntesis de antocianinas, y en consecuencia, el desarrollo de color en los frutos, lo que afectó la calidad (Ozcan et al., 2016). Con respecto a los aminoácidos, el triptófano $(15 \mathrm{mg}$ $\mathrm{L}^{-1}$ ) incrementa el contenido de clorofila (a y b) y el ácido glutámico (500 a $1500 \mathrm{mg} \mathrm{L}^{-1}$ ) favorece el color rojo en los frutos y promueve o incrementa las antocianinas en lichi (Zeng et al., 2012). El color verde en las zonas cercanas al pedúnculo de los higos da la apariencia de inmadurez, a pesar de haber sido cosechados en madurez de consumo, lo que conlleva a una baja preferencia por parte del consumidor; además, los tratamientos que adelantaron la cosecha de los frutos (T1, T2, T3, y T5) pierden esta ventaja económica al considerar el efecto negativo del color.

\section{Firmeza}

Los frutos que recibieron el T2, cosechados el 15 de mayo, presentaron mayor firmeza que los de T4 (Cuadro 3). Por otro lado, los higos de T1 y T2 cosechados el 15 de junio fueron más firmes con respecto a los de T3 y T4. En los frutos de la tercera cosecha no se presentaron diferencias estadísticas significativas. García-Ruiz et al. (2013) reportaron que los frutos del cv. Netzahualcóyotl son suaves, sin indicar valores de firmeza. Los resultados en esta investigación indican que la combinación de TDZ con $\mathrm{AG}_{3}$ favorece la firmeza de los frutos de higo Netzahualcóyotl, lo que coincide con lo reportado en uvas de mesa por Jung et al. (2015), donde la aplicación de $T D Z$ y $\mathrm{AG}_{3}$ incrementaron la firmeza. El calcio es un elemento clave en la preservación de la firmeza en los frutos y de acuerdo con Nisler (2018), el TDZ influye en las concentraciones de $\mathrm{Ca}$ inter e intracelular. Por otro lado, Crisosto et al. (2010) mencionaron que valores altos de firmeza y sólidos solubles en higo fresco son deseables para frutos en estado avanzado de madurez porque toleran la cosecha y el manejo postcosecha; por lo tanto, los frutos de los tratamientos T1 y T2 presentaron mayor vida de anaquel.

\section{Sólidos solubles totales (SST) y acidez titulable (AT)}

El Cuadro 3 muestra que los tratamientos aplicados a las higueras cv. Netzahualcóyotl incrementaron significativamente los SST en los frutos cosechados el 15 de junio con respecto al testigo (T6). Los valores de SST del testigo (T6) coincidieron con los obtenidos por Mendoza-Castillo et al. (2017) para el mismo cultivar. Sustancias como el TDZ (Jung et al., 2015), $\mathrm{AG}_{3}$ (Erogul y Sen, 2015) y triptófano (El Sayed et al., 2014) favorecen los SST en frutales. Estos incrementos se asocian con la rápida transformación metabólica de compuestos 


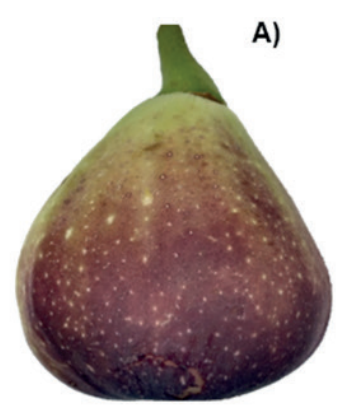

D)

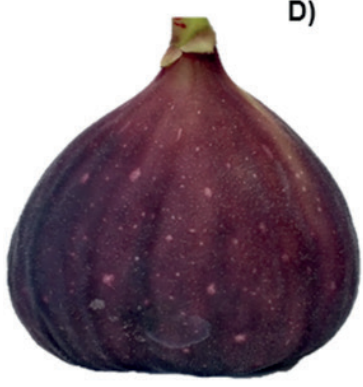

B)

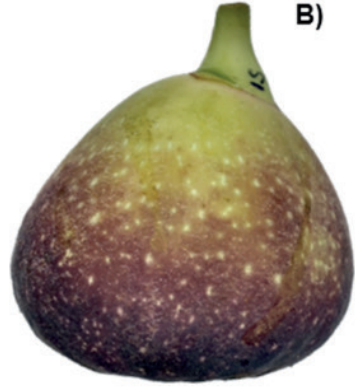

E)

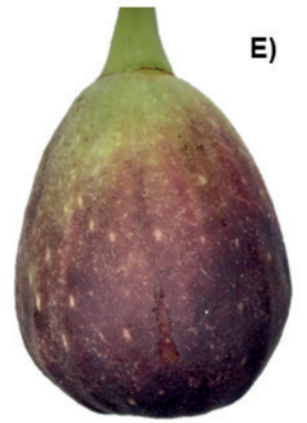

C)
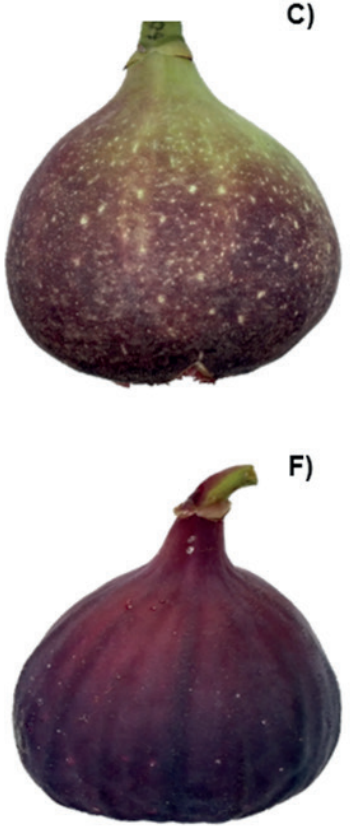

Figura 2. Color de los higos cv. Netzahualcóyotl cosechados en madurez de consumo. A): T1 (100 mg L-1 TDZ y 50 mg L-1 $\mathrm{AG}_{3}$ ), B): T2 (100 mg L-1 TDZ , $25 \mathrm{mg} \mathrm{L}^{-1} \mathrm{AG}_{3}$ y $150 \mathrm{mg} \mathrm{L}^{-1}$ triptófano), C): T3 (50 mg L-1 TDZ, $25 \mathrm{mg} \mathrm{L}^{-1} \mathrm{AG}_{3}$ y $150 \mathrm{mg} \mathrm{L}^{-1}$ ácido glutámico), D): T4 (150 mg L-1 ácido glutámico y $100 \mathrm{mg} \mathrm{L}^{-1}$ tiamina), E): T5 (25 mg L-1 TDZ, $150 \mathrm{mg} \mathrm{L}^{-1}$ triptófano y $150 \mathrm{mg} \mathrm{L}^{-1}$ ácido glutámico) y F): T6 (testigo, sin aplicación).

Cuadro 3. Sólidos solubles totales (SST), acidez titulable (AT) y firmeza (N) de los frutos de higo cv. Netzahualcóyotl tratados con biorreguladores, cosechados en diferentes fechas durante 2017.

\begin{tabular}{|c|c|c|c|c|c|c|c|c|c|}
\hline \multirow[b]{2}{*}{ Tratamiento } & \multicolumn{3}{|c|}{15 de mayo } & \multicolumn{3}{|c|}{15 de junio } & \multicolumn{3}{|c|}{15 de julio } \\
\hline & $\begin{array}{c}\text { SST } \\
\text { ('Brix) }\end{array}$ & AT (\%) & $\begin{array}{c}\text { Firmeza } \\
(\mathrm{N})\end{array}$ & $\begin{array}{c}\text { SST } \\
\text { ( }{ }^{\circ} \text { Brix) }\end{array}$ & AT $(\%)$ & $\begin{array}{c}\text { Firmeza } \\
(\mathrm{N})\end{array}$ & $\begin{array}{c}\text { SST } \\
\left({ }^{\circ} \text { Brix }\right)\end{array}$ & AT (\%) & $\begin{array}{c}\text { Firmeza } \\
(\mathrm{N})\end{array}$ \\
\hline T1 & $25.9 a$ & $0.26 a$ & $0.20 a b c$ & $22.6 \mathrm{a}$ & $0.24 \mathrm{a}$ & $0.80 \mathrm{a}$ & $21.0 \mathrm{a}$ & $0.29 a b$ & $0.35 a$ \\
\hline $\mathrm{T} 2$ & $24.1 \mathrm{a}$ & $0.26 a$ & $0.34 \mathrm{a}$ & $22.7 \mathrm{a}$ & $0.23 a$ & $0.89 a$ & 19.9 a & $0.20 b$ & $0.28 \mathrm{a}$ \\
\hline T3 & $24.8 \mathrm{a}$ & $0.22 \mathrm{a}$ & $0.31 a b$ & $23.1 \mathrm{a}$ & $0.30 \mathrm{a}$ & $0.34 \mathrm{c}$ & $21.3 a$ & $0.27 a b$ & $0.29 a$ \\
\hline $\mathrm{T} 4$ & $25.1 \mathrm{a}$ & $0.29 a$ & $0.14 \mathrm{c}$ & $23.2 \mathrm{a}$ & $0.28 a$ & $0.34 \mathrm{c}$ & $19.0 \mathrm{a}$ & $0.34 \mathrm{a}$ & $0.31 \mathrm{a}$ \\
\hline T5 & $22.8 \mathrm{a}$ & $0.26 a$ & $0.17 b c$ & $27.2 \mathrm{a}$ & $0.33 a$ & $0.56 b$ & $20.8 \mathrm{a}$ & $0.30 \mathrm{ab}$ & $0.25 a$ \\
\hline T6 & $23.2 \mathrm{a}$ & $0.29 a$ & $0.17 b c$ & $17.3 b$ & $0.31 \mathrm{a}$ & $0.35 b c$ & $18.9 \mathrm{a}$ & $0.30 a b$ & $0.26 a$ \\
\hline CV (\%) & 12.4 & 23.3 & 38.9 & 8.0 & 16.9 & 17.6 & 10.5 & 17.5 & 19.9 \\
\hline
\end{tabular}

Medias con letras iguales en las columnas no son estadísticamente diferentes (Tukey, $\mathrm{P} \leq 0.05)$. T1 (100 mg L-1 TDZ y $50 \mathrm{mg} \mathrm{L}^{-1} \mathrm{AG}_{3}$ ), T2 (100 mg L-1 TDZ , $25 \mathrm{mg} \mathrm{L}^{-1} \mathrm{AG}_{3}$ y $150 \mathrm{mg} \mathrm{L}^{-1}$ triptófano), T3 (50 mg L-1 TDZ, $25 \mathrm{mg} \mathrm{L}^{-1} \mathrm{AG}_{3}$ y $150 \mathrm{mg} \mathrm{L}^{-1}$ ácido glutámico), T4 (150 mg L-1 ácido glutámico y 100 $\mathrm{mg} \mathrm{L}^{-1}$ tiamina), $5^{3}$ (25 $\mathrm{mg} \mathrm{L}^{-1} \mathrm{TDZ}, 150 \mathrm{mg} \mathrm{L}^{-1}$ triptófano y $150 \mathrm{mg} \mathrm{L}^{-1}$ ácido glutámico) y T6 (testigo, sin aplicación). CV: coeficiente de variación.

solubles y mayor cantidad de polisacáridos que se convierten en azúcares reductores (Krishna et al., 2012). Esta variable mejora conforme la maduración de los higos avanza e indica la aceptabilidad de los frutos (Crisosto et al., 2010). En relación con la AT, T4 incrementó el contenido de ácido cítrico en los frutos de la cosecha del 15 de julio, comparado con T2. Se ha reportado que la aplicación de ácido glutámico incrementa la acidez en frutos de lima mexicana (Ariza et al., 2015). Los valores de AT del testigo (T6) coinciden con los reportados por Crisosto et al. (2010) en higos madurados en el árbol. 


\section{CONCLUSIONES}

La aplicación dirigida de biorreguladores en higo cv. Netzahualcóyotl estimula la brotación de yemas secundarias vegetativas y reproductivas; sin embargo, ocasiona el aborto en ambos casos. La aplicación de TDZ mezclado con $A_{3}$; TDZ más $A_{3}$ y triptófano; TDZ combinado con $\mathrm{AG}_{3}$ y ácido glutámico y una mezcla de TDZ con triptófano y ácido glutámico adelantaron 15 d la cosecha de los higos desarrollados de la yema principal. La aplicación de los biorreguladores mejoró las variables de calidad del fruto de la yema principal como tamaño (diámetro y longitud), peso individual, firmeza, AT y SST; no obstante, afectó el color en forma negativa y no se incrementó el rendimiento por planta. Los tratamientos con biorreguladores mejoraron algunas variables de calidad, pero ninguno en particular fue consistente durante la producción.

\section{BIBLIOGRAFÍA}

AOAC, Association of Official Analytical Chemists (1990) Official Methods of Analysis. 15th edition. Association of Official Analytical Chemists. Arlington, Virginia. USA.

Ariza F. R., A. Barrios A., M. Herrera G., F. Barbosa M., A. Michel A., M. A. Otero S. e I. Alia T. (2015) Fitohormonas y bioestimulantes para la floración, producción y calidad de lima mexicana de invierno. Revista Mexicana de Ciencias Agrícolas 6:1653-1666.

Bahar A. and A. Lichter (2018) Effect of controlled atmosphere on the storage potential of Ottomanit fig fruit. Scientia Horticulturae 227:196-201, https://doi.org/10.1016/j.scienta.2017.09.036

Belal B. E. A., M. A. El-Kenawy and M. K. Uwakiem (2016) Foliar application of some amino acids and vitamins to improve growth, physical and chemical properties of flame seedless grapevines. Egyptian Journal of Horticulture 43:123-136.

Calderón-Zavala G. and J. Rodríguez-Alcázar (2000) Thidiazuron (n-phenil-n1-(1, 2, 3-thidiazol-5-yl) urea) as a promoter of budbreak on peach (Prunus persica L. Batsch) and japanese plum (Prunus salicina Lindl.). Revista Chapingo Serie Horticultura 6:117-120, https://doi.org/10.5154/r.rchsh.1999.02.014

Cao Y. P., Z. K. Gao., J. T. Li., G. H. Xu and M. Wang (2010) Effects of extraneous glutamic acid on nitrate contents and quality of chinese chive. Acta Horticulturae 856:91-98, https://doi.org/10.17660/ActaHortic.2010.856.11

Colla G. and Y. Rouphael (2015) Biostimulants in horticulture. Scientia Horticulturae 196:1-2, https://doi.org/10.1016/j.scienta.2015.10.044

Crisosto C. H., V. Bremer, L. Ferguson and G. M. Crisosto (2010) Evaluating quality attributes of four fresh fig (Ficus carica L.) cultivars harvested at two maturity stages. HortScience 45:707-710, https://doi.org/10.21273/HORTSCI.45.4.707

du Jardin P. (2015) Plant biostimulants: definition, concept, main categories and regulation. Scientia Horticulturae 196:3-14, https://doi.org/10.1016/j.scienta.2015.09.021

El Sayed O. M., O. H. M. El Gammal and A. S. M. Salama (2014) Effect of proline and tryptophan amino acids on yield and fruit quality of Manfalouty pomegranate variety. Scientia Horticulturae 169:15, https://doi.org/10.1016/j.scienta.2014.01.023

Erogul D. and F. Sen (2015) Effects of gibberellic acid treatments on fruit thinning and fruit quality in Japanese plum (Prunus salicina Lindl). Scientia Horticulturae 186:137-142, https://doi.org/10.1016/j.scienta.2015.02.019

Gao Z., M. Hu., M. Zhu., M. Li., J. Wen., P. Fan... and C. Zhao (2017) Effects of pre-harvest $\mathrm{GA}_{3}$ spraying on yield, quality and storability of mango fruit. Journal of Fruit Science 34:744-751.

García M. R., A. López J., C. Saucedo V., S. Salazar-García y J. Suárez E. (2015) Maduración y calidad de frutos de mango 'Kent' con tres niveles de fertilización. Revista Mexicana de Ciencias Agrícolas 6:665-678, https://doi.org/10.29312/remexca.v6i4.610

García-Ruiz M. T., V. M. Mendoza-Castillo, E. Valadez-Moctezuma and A. Muratalla-Lúa (2013) Initial assessment of natural diversity in Mexican fig landraces. Genetics and Molecular Research 12:3931-3943, https://doi.org/10.4238/2013.September.23.12

Goyer A. (2010) Thiamine in plants: aspects of its metabolism and functions. Phytochemistry 71:1615-1624, https://doi org/10.1016/j.phytochem.2010.06.022

Hawerroth F. J., F. G. Herter, J. C. Fachinello, J. L. Petri, M. E. Prezotto, L. B. Haas and A. Pretto (2011) Aumento da produção de pereira asiática pelo uso de fitorreguladores. Ciência Rural 41:17501754.

Ikegami H., H. Nogata, Y. Inoue, S. Himeno, H. Yakushiji, C. Hirata... and T. Nakahara (2013) Expression of FCFT1, a FLOWERING LOCUS T-like gene, is regulated by light and associated with inflorescence differentiation in fig (Ficus carica L.). BCM Plant Biology 13:216, https://doi.org/10.1186/1471-2229-13-216

Jung M. H., B. H. Lee, Y. S. Park, J. P. Oh, H. S. Kim and H. S. Park (2015) Characteristics of the fruits and flesh softening delay induced by $\mathrm{GA}_{3}$ and thidiazuron (TDZ) treatment in 'Heukboseok' grape. Korean Journal of Horticultural Science and Technology 33:186-195, https://doi.org/10.7235/hort.2015.14100

Krishna H., B. Das, B. L. Attri, A. Kumar and N. Ahmed (2012) Interaction between different pre- and postharvest treatments on shelf life extension of 'Oregon Spur' apple. Fruits 67:31-40, https://doi. org/10.1051/fruits/2011064

Kurubar A. R., T. B. Alloli, M. K. Naik and S. G. Angadi (2017) Effects of gibberellic acid on growth, yield and fruit quality of fig (Ficus carica L.). Acta Horticulturae 1173:183-188, https://doi. org/10.17660/ActaHortic.2017.1173.31

Li Y. H., Y. J. Wu, B. Wu, M. H. Zou, Z. Zhang and G. M. Sun (2011) Exogenous gibberellic acid increases the fruit weight of 'Comte de Paris' pineapple by enlarging flesh cells without negative effects on fruit quality. Acta Physiologiae Plantarum 33:1715-1722 https://doi.org/10.1007/s11738-010-0708-2

Macías R. H., M. M. Villa C., A. Muñoz V., M. A. Velásquez V., M. Rivera G. y M. C. Potisek T. (2013) Enraizamiento y brotación de vareta de higuera en contenedores de plástico cerrados: resultados preliminares. AGROFAZ 13:37-44

Melgarejo M. P. (2000) Tratado de Fruticultura para Zonas Áridas y Semiáridas. Vol. I. El Medio Ecológico, la Higuera, el Alcaparro y el Nopal. Ediciones Mundi-Prensa. Madrid, España. 382 p.

Mendoza-Castillo V. M., J. M. Vargas-Canales, G. Calderón-Zavala, M. C. Mendoza-Castillo and A. Santacruz-Varela (2017) Intensive production systems of fig (Ficus carica L.) under greenhouse conditions. Experimental Agriculture 53:339-350, https://doi org/10.1017/S0014479716000405

Milić B., J. Tarlanović, Z. Keserović, N. Magazin, M. Miodragović and G. Popara (2018) Bioregulators can improve fruit size, yield and plant growth of northern highbush blueberry (Vaccinium corymbosum L.). Scientia Horticulturae 235:214-220, https:// doi.org/10.1016/j.scienta.2018.03.004

Muñoz V. J. A., M. Palomo R., H. Macías R., M. Rivera G. y G. Esquivel A. (2015) Dinámica del crecimiento fenológico de higuera (Ficus carica L.) con altas densidades de plantación en macro-túneles. AGROFAZ 15:133-141

Mustafa A., M. Imran, M. Ashraf and K. Mahmood (2018) Perspectives of using L-tryptophan for improving productivity of agricultural crops: a review. Pedosphere 28:16-34, https://doi.org/10.1016/ S1002-0160(18)60002-5

Nisler J. (2018) TDZ: mode of action, use and potential in agriculture. In: Thidiazuron: From Urea Derivative to Plant Growth Regulator. N. Ahmad and M. Faisal (eds.). Springer. Singapore. pp:37-60 https://doi.org/10.1007/978-981-10-8004-3_2

Osuna-Enciso T., Z. M. Chavarín-Navarro, J. A. Carrillo-Fasio, J. B. ValdezTorres, J. Basilio-Heredia, M. A. Báez-Sañudo, S. HernándezVerdugo y J. M. Osuna-Rodríguez (2019) Efecto de aspersiones de biorreguladores en precosecha sobre el crecimiento y maduración del mango Keitt. Revista Fitotecnia Mexicana 42:259-268, https://doi.org/10.35196/rfm.2019.3.259-268

Ozcan Y., M. Ucar., K. Yildiz and B. Ozturk (2016) Pre-harvest gibberellic acid $\left(\mathrm{GA}_{3}\right)$ treatments play an important role on bioactive compounds 
and fruit quality of sweet cherry cultivars. Scientia Horticulturae 211:358-362, https://doi.org/10.1016/j.scienta.2016.09.019

Pereira C., M. J. Serradilla, F. Pérez-Gragera, A. Martín, M. C. Villalobos and M. López-Corrales (2017) Evaluation of agronomic and fruit quality traits of fig tree varieties (Ficus carica L.) grown in Mediterranean conditions. Spanish Journal of Agricultural Research. 15:e0903, https://doi.org/10.5424/sjar/2017153-10403

Rodrigues A. C., J. C. Fachinello and J. B. da Silva (1997) Antecipação e uniformização da maturação de figos cv. Roxo de Valinhos com uso de fitoreguladores e óleo de oliva. Revista Brasileira de Agrociência 3:69-73.

Sadak M. S. H., M. T. Abdelhamid and U. Schmidhalter (2015) Effect of foliar application of aminoacids on plant yield and some physiological parameters in bean plants irrigated with seawater. Acta Biológica Colombiana 20:141-152, https://doi.org/10.15446/ abc.v20n1.42865

SAS Institute (2004) SAS/STAT® 9.1 User's Guide. SAS Institute. Cary, North Carolina, USA. 5121 p.

Souza E. R., M. C. Pereira, L. S. Santos, V. G. Ribeiro, J. A. A. Pionório e E. A. Araújo (2010) Qualidade da uva 'Superior Seedless' com aplicações de benziladenina combinadas ou não com ácido giberélico. Revista Caatinga 23:144-148.

Weiss D. and N. Ori (2007) Mechanisms of cross talk between gibberellin and other hormones. Plant Physiology 144:1240-1246, https:// doi.org/10.1104/pp.107.100370

Wójcik P., A. Skorupińska and H. Gubbuk (2016) Impacts of pre- and postbloom sprays of tryptophan on calcium distribution within 'Red Jonaprince' apple trees and on fruit quality. HortScience 51:1511-1516, https://doi.org/10.21273/HORTSCI11216-16

Yemiş 0., E. Bakkalbașı and N. Artık (2012) Changes in pigment profile and surface colour of fig (Ficus carica L.) during drying. International Journal of Food Science and Technology 47:1710-1719, https:// doi.org/10.1111/j.1365-2621.2012.03025.x

Yu C., D. G. Lv, S. J. Qin, L. Yang., H. Y. Ma and G. C. Liu (2010) Changes in photosynthesis, fluorescence, and nitrogen metabolism of hawthorn (Crataegus pinnatifida) in response to exogenous glutamic acid. Photosynthetica 48:339-347, https://doi org/10.1007/s11099-010-0044-1

Zang Y. X., I. J. Chun, L. L. Zhang, S. B. Hong, W. W. Zheng and K. Xu (2016) Effect of gibberellic acid application on plant growth attributes, return bloom, and fruit quality of rabbiteye blueberry. Scientia Horticulturae 200:13-18, https://doi.org/10.1016/j. scienta.2015.12.057

Zeng L. D., J. L. Liao and H. B. Chen (2012) Effects of glutamic acid and TDZ (Thidiazuron) on the fruit coloration and quality of Litchi chinensis Sonn. Journal of Tropical and Subtropical Botany 20:382-387, https://doi.org/10.3969/j.issn.10053395.2012.04.010

Zermeño A., J. A. Gil, A. Hernández, R. Rodríguez, H. Ramírez, A. Benavides... y L. Ibarra (2010) Efectos del encalado completo y aplicación de TDZ sobre la brotación, rendimiento y calidad de frutos del manzano cv. Golden Delicious. Bioagro 22:75-80. 